\title{
Biomechanical Comparison of Different External Fixation Configurations for Posttraumatic Pelvic Ring Instability
}

\author{
Simon Tiziani, ${ }^{1}$ Georg Osterhoff, ${ }^{1}$ Stephen J. Ferguson, ${ }^{2}$ \\ Gregor Spreiter, ${ }^{2}$ Max J. Scheyerer, ${ }^{1}$ Gian-Leza Spinas, ${ }^{1}$ Guido A. Wanner, ${ }^{1}$ \\ Hans-Peter Simmen, ${ }^{1}$ and Clément M. L. Werner ${ }^{1}$ \\ ${ }^{1}$ Department of Surgery, Division of Trauma Surgery, University Hospital Zurich, Raemistrasse 100, 8091 Zurich, Switzerland \\ ${ }^{2}$ Institute for Biomechanics, ETH Zurich, HCI-E355.2 Wolfgang-Pauli-Strasse 10, 8093 Zurich, Switzerland
}

Correspondence should be addressed to Simon Tiziani; simon.tiziani@uzh.ch

Received 10 September 2013; Accepted 13 November 2013; Published 2 February 2014

Academic Editor: Jörn Kircher

Copyright (C) 2014 Simon Tiziani et al. This is an open access article distributed under the Creative Commons Attribution License, which permits unrestricted use, distribution, and reproduction in any medium, provided the original work is properly cited.

Background. External fixation is useful in the primary treatment of pelvic ring injuries. The present study compared the biomechanical stability of five different configurations of an external pelvic ring fixation system. Methods. Five configurations of an anterior external pelvic ring fixation system were tested using a universal testing machine. One single connecting rod was used in group "SINGLE," two parallel connecting rods in group "DOUBLE," two and four rods, respectively, in a tent-like configuration in groups "SINGLE TENT" and "DOUBLE TENT," and a rhomboid-like configuration in group "RHOMBOID." Each specimen was subjected to a total of 2000 consecutive cyclic loadings at $1 \mathrm{~Hz}$ lateral compression/distraction $( \pm 50 \mathrm{~N})$ and torque $( \pm 0.5 \mathrm{Nm})$ loading alternating every 200 cycles. Translational and rotational stiffness were determined at 100, 300, 500, 700, and 900 cycles. Results. The "SINGLE TENT" and "RHOMBOID" configurations already failed with a preloading of $50 \mathrm{~N}$ compression force. The "DOUBLE" configuration had around twice the translational stability compared with the "SINGLE" and "DOUBLE TENT" configurations. Rotational stiffness observed for the "DOUBLE" and "DOUBLE TENT" configurations was about 50\% higher compared to the SINGLE configuration. Conclusion. Using two parallel connecting rods provides the highest translational and rotational stability.

\section{Background}

Although unstable pelvic ring injuries are relatively rare [1], patients suffering from such injuries often show extensive haemorrhage [2]. Blood loss can occur from osseous structures at the fracture site, venous bleeding from the sacral plexus, or arterial bleeding [3]. Pelvic volume increases with pelvic ring disruption, which further hinders haemostasis. The primary objective in such situations is to reestablish pelvic ring integrity and stability, reducing pelvic volume in the process $[3,4]$. Together with pelvic packing and clamping, external pelvic fixation has become an established adjunct for stabilizing unstable fractures and increasing chances of haemostasis [5-10]. External fixation may assist haemostasis in different ways, reducing fracture surfaces, ensuring blood clot stability, stopping venous bleeding, and achieving some tamponade by reducing pelvic volume [11, 12], though it has been shown that it may not induce pressure-induced tamponade [6]. There are numerous different external fixator constructs with each making different configurations possible. Furthermore there are different locations for pin anchoring in the pelvis. A supra-acetabular placement of the external fixator pins has been shown to be superior in stability compared to pins placed in the iliac crest $[13,14]$. Continuing stability of the external fixation is crucial in ensuring optimal chance for haemostasis. Patients rarely present with isolated injuries to the pelvis. Concomitant abdominal injuries are frequent, making, for example, further abdominal surgery necessary [15]. This and the fact that some patients are obese, have made it necessary to come up with different configurations of the external fixation construct to fit the respective situations. The following configurations, tested in this study, were in use at 


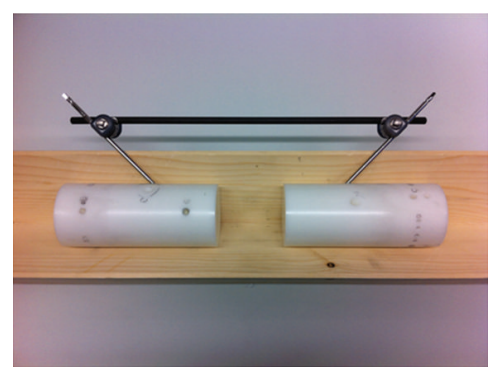

(a)

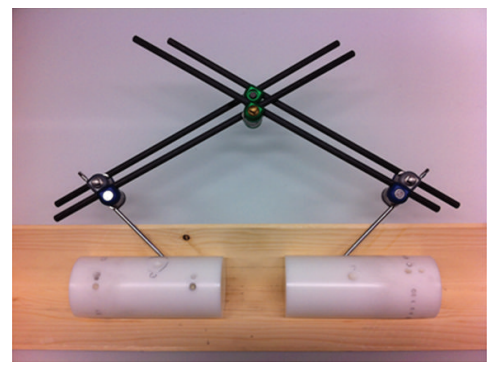

(d)

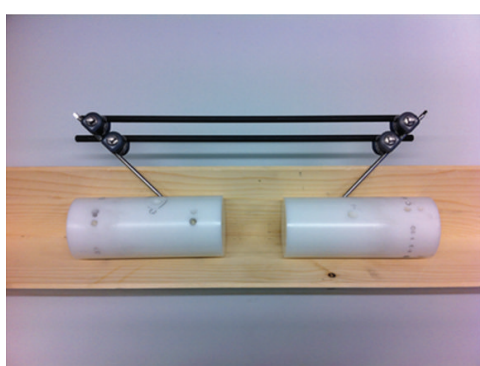

(b)

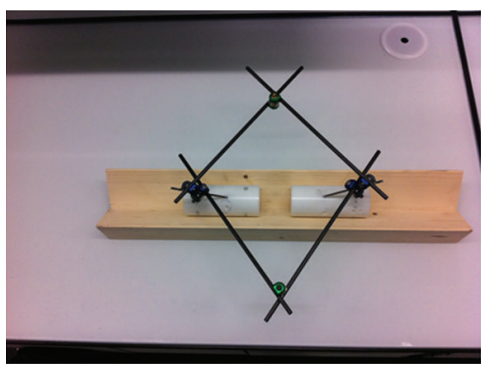

(e)

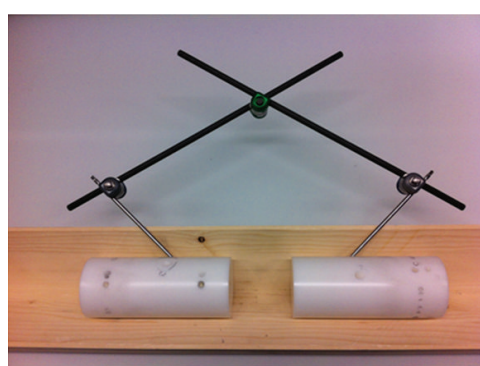

(c)

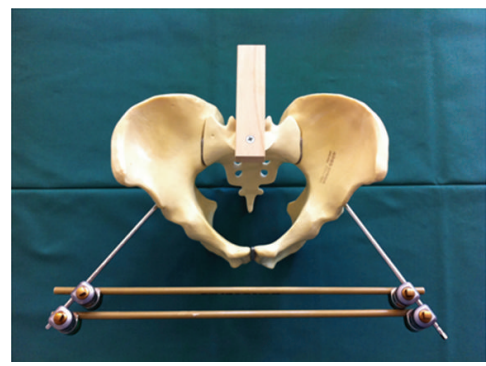

(f)

FIGURE 1: Configurations and pelvic instrumentation. (a) The "SINGLE" configuration. (b) The "DOUBLE" configuration. (c) The "SINGLETENT" configuration. (d) The "DOUBLE-TENT" configuration. (e) The "RHOMBOID" configuration. (f) A "DOUBLE" configuration mounted on a synthetic Synbone pelvis.

our University Hospital when treating unstable pelvic ring fractures. The "SINGLE" (Figure 1(a)) and "DOUBLE" (Figure 1(b)) configurations represent standard configurations. The "SINGLE TENT" (Figure 1(c)) and "DOUBLE TENT" (Figure 1(d)) configurations are used where the distance from the symphysis to the skin is increased in obese patients, making the application of the "SINGLE" and "DOUBLE" configurations impossible. Finally the "RHOMBOID" (Figure 1(e)) configuration is installed when further abdominal procedures (e.g., laparotomy) are to be expected.

The aim of the study was to compare the stability of five different configurations of the same external pelvic fixation construct. The hypothesis was that there would be no difference in stability between the different configurations.

\section{Materials and Methods}

2.1. Samples. The testing protocol included the testing of five different configurations of the same external fixation construct for the pelvis (Hoffmann II, Stryker, Kalamazoo, MI, USA). The groups to be tested included the following configurations: "SINGLE" (Figure 1(a)), "DOUBLE" (Figure 1(b)), "SINGLE TENT" (Figure 1(c)), "DOUBLE TENT" (Figure $1(\mathrm{~d})$ ), and "RHOMBOID" (Figure 1(e)). The fixator's pins (diameter $5 \mathrm{~mm}$ ) were inserted into polyoxymethylene cylinders (diameter $70 \mathrm{~mm}$ ) (Delrin, DuPont, Wilmington, DE, USA), one for each pin. Distance between the two polyoxymethylene cylinders and angle of pin insertion were measured at an external fixation device mounted in the supra-acetabular region of a synthetic model of the pelvis (Pelvis, Synbone, Malans, Switzerland) (Figure 1(f)). The pins were than screwed into the polyoxymethylene cylinders at a 45 degree angle and $7 \mathrm{~cm}$ from the later inward facing edge, making the distance from pin entry to pin entry site $21 \mathrm{~cm}$. The couplings interfacing the pins and the rods were always placed with their upper edge on the first mark on the pins. In the "TENT" and "DOUBLE-TENT" configuration the distance between the pin-rod interface and the rod-rod interface was $24 \mathrm{~cm}$ (in the "DOUBLE-TENT" configuration this refers to the outer tent), resulting in a distance from rod-rod coupling to the surface of the cylinders of $25 \mathrm{~cm}$ in the plumb line. The distance from rod-pin interface to the rod-rod interface in the "RHOMBOID" configuration was $26 \mathrm{~cm}$ on all sides. The shapes of the different configurations were determined by an experienced orthopedic trauma surgeon (C.W). The five different configurations were attached to the pins via the rodpin interface couplings, which all were tightened by the same person (S.T).

2.2. Biomechanical Testing. For the tests two different loading scenarios were selected. Firstly a translational loading scenario was applied, where the construct was loaded simulating compression and distraction in the pelvis. Secondly a rotational loading scenario was applied that would simulate, for example, hip bending. These two scenarios were chosen because they address the most common situations occurring in the postop phase: patients in supine position, leg movement, and patient transfer.

Thus all samples were tested using a universal testing machine (ElectroPulse E10000, Instron, Norwood, MA, USA) (Figure 2) according to the following protocol: alternating cycles of compression and distraction (along the cylindrical axis) and rotational loading (rotation around the cylindrical axis). Each sequence consisting of 200 cycles at $1 \mathrm{~Hz}$ was 


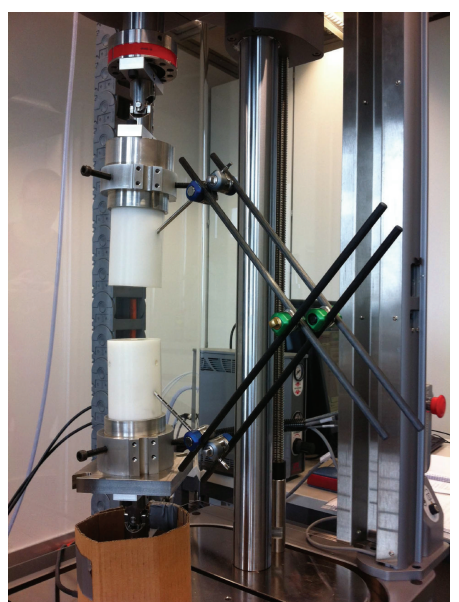

FIgURE 2: Experimental setup. Two polyoxymethylene cylinders instrumented with a Hoffmann II external fixator in the "DOUBLETENT" configuration mounted on the Intron testing machine. Testing included compression/distraction and rotational loading.

run 5 times, making a total of 2000 cycles (1000 cycles each) per sample.

After slowly loading and observing a displacement of $>25 \mathrm{~mm}$ with $\pm 50 \mathrm{~N}$ translational load, $50 \mathrm{~N}$ was chosen for loading the cycles in translational loading. Rotational torque was set at $\pm 5 \mathrm{Nm}$.

Measurements during testing were recorded by a $\pm 1000 \mathrm{~N}$ (compression/distraction forces) and a $25 \mathrm{Nm}$ (torque forces) loading cell and the computer programme calculated translational and rotational stiffness. Failure, as defined in the testing protocol, leading to abort of testing included: displacement overshooting the machine's range of motion $( \pm 30 \mathrm{~mm}$ translation and \pm 135 degrees rotation) and/or interface or construct failure.

\subsubsection{Evaluation}

Translational Stiffness [N/mm]. Translational stiffness readings were taken at 100,300,500,700, and 900 cycles to be analysed statistically. This is a measure for resisting displacement when loaded simulating lateral compression and distraction.

Rotational Stiffness [Nm/degree]. Rotational stiffness readings were taken at 100,300,500, 700, and 900 cycles to be analysed statistically. This is a measure for resisting torque forces on the pelvis.

2.3. Statistical Analysis. After preliminary testing, a sample size calculation was performed using PS power and sample size calculations 3.0 (alpha error: 0.05) [16].

With an expected difference in means of $0.5 \mathrm{~N} / \mathrm{mm}$ and a standard deviation of $0.15 \mathrm{~N} / \mathrm{mm}$ for translational stiffness and an expected difference in means of $0.08 \mathrm{Nm} /$ degree and a standard deviation of $0.025 \mathrm{Nm} /$ degree for rotational stiffness the calculated number of samples to be able to reject the null hypothesis that the population means of the experimental

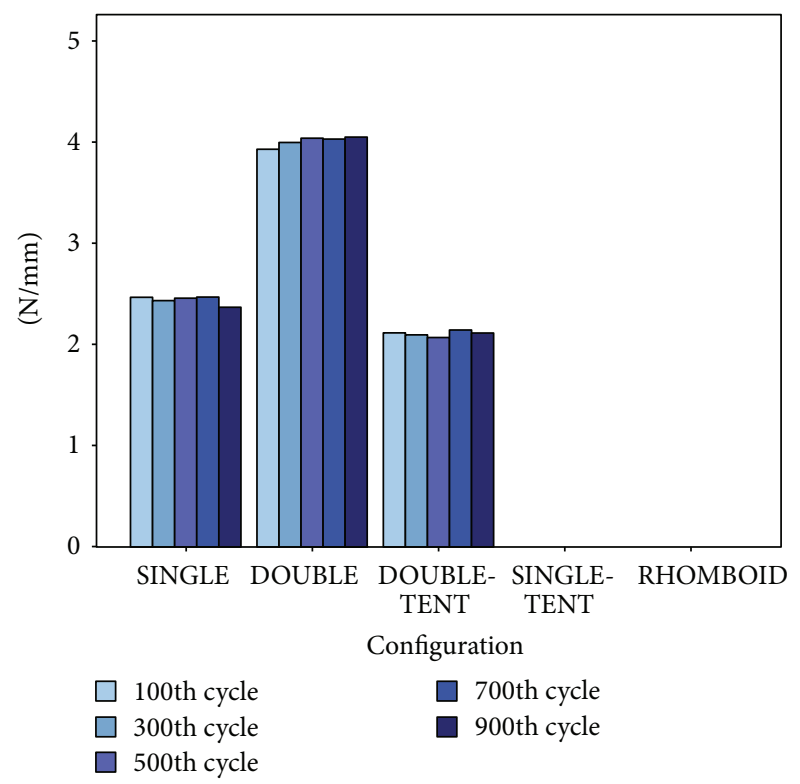

FIGURE 3: Translational stiffness. Results for translational stiffness of the five different configurations at 100, 300, 500, 700, and 900 cycles. The "SINGLE-TENT" and the "RHOMBOID" configurations failed and thus yielded no results.

and control groups are equal with a probability (power) of 0.8 was 3 per group.

Comparison of translational and rotational stiffness was done using a one-way ANOVA with a post hoc Bonferroni adjustment for multiple comparisons using SPSS for Windows V20.0 (SPSS, Chicago, Illinois, USA). Differences were considered significant for values of $P<0.05$.

\section{Results}

The "SINGLE TENT" and the "RHOMBOID" configurations showed more than $30 \mathrm{~mm}$ displacement before reaching $50 \mathrm{~N}$ lateral compression-distraction loading, resulting in failure as defined by the testing protocol.

3.1. Translational Stiffness (Figure 3). The "DOUBLE" configuration was $59 \%-71 \%$ stiffer than the "SINGLE" configuration at $100,300,500,700$, and 900 cycles $(P=.002, .003, .005$, .001 , and .001). Comparing it to the "DOUBLE TENT" configuration, the "DOUBLE" configuration was between $86 \%$ and $95 \%$ stiffer $(P=.001, .001, .001, .001$, and .001$)$. There was no significant difference in translational stiffness between the "DOUBLE TENT" and the "SINGLE" configuration.

3.2. Rotational Stiffness (Figure 4). The "DOUBLE" configuration exceeded the "SINGLE" configuration in stiffness by $35 \%$ to $60 \%$ at $100,300,500,700$, and 900 cycles $(P=.025$, $.019, .031, .003$, and .004). The "DOUBLE TENT" configuration was stiffer than the SINGLE configuration at 100 cycles (41\%, $P=.012), 500$ cycles $(43 \%, P=.011), 700$ cycles $(57 \%$, $P=.005)$, and 900 cycles $(55 \%, P=.006)$; there was no 


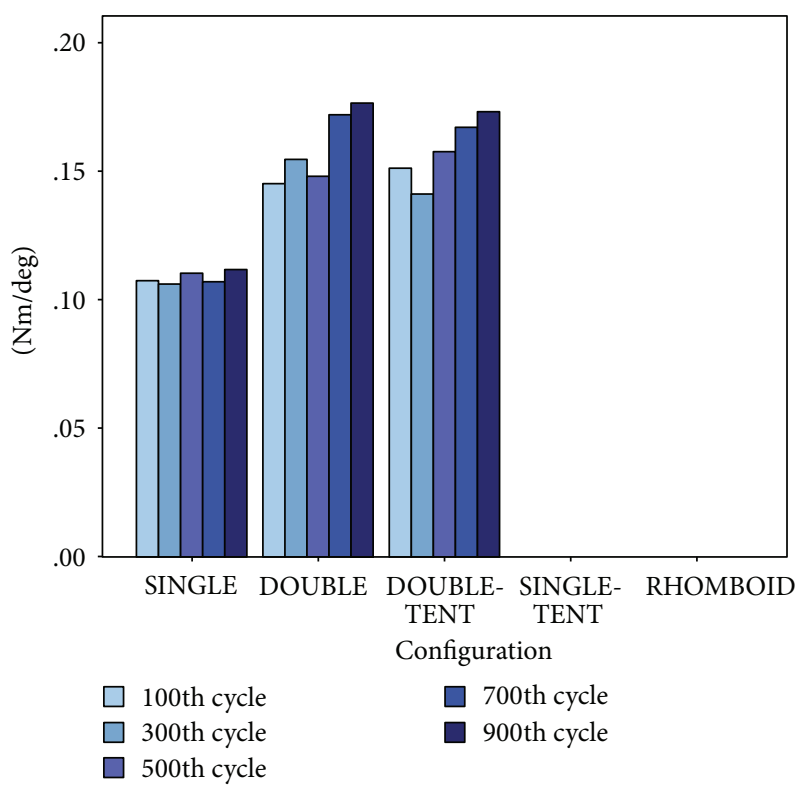

FIGURE 4: Rotational stiffness. Results for rotational stiffness of the five different configurations at 100, 300, 500, 700, and 900 cycles. The "SINGLE-TENT" and the "RHOMBOID" configurations failed and thus yielded no results.

significant difference at 300 cycles. Also, there was no significant difference between the "DOUBLE" and the "DOUBLE TENT" configurations.

No permanent deformation was observed in any of the tested implants. No interface failure occurred.

\section{Discussion}

The aim of the study was to compare different configurations of the same external pelvic fixation construct, testing them for translational and rotational stiffness. The "SINGLE" configuration showed two-third of the "DOUBLE" configuration's stiffness and the "DOUBLE" configuration was twice as stiff as the "DOUBLE TENT" configuration in terms of translational stiffness. There was no significant difference between the "DOUBLE" and the "DOUBLE TENT" configurations regarding rotational stiffness. Between the "DOUBLE TENT" and "SINGLE" configuration there was a significant difference in rotational stiffness and the "DOUBLE TENT" was twice as stiff. Both the "RHOMBOID" and the "SINGLE TENT" configurations failed.

In the beginning there were efforts to devise external fixator constructs that would by themselves stabilize vertically unstable pelvic fractures. This was demonstrated not to be possible $[17,18]$. As soon as this was realized, complexity in external fixation construct decreased, seeing as multiple pins increase the chance for pin-tract infections [19]. Research has since been focused on finding the optimal pin placement site $[13,20]$ or determining difference in stability between different fixator constructs and configurations [21-24]. It was not clear how our fixation construct's stability would change when the configuration was adapted for obese patients or patients undergoing abdominal surgery.
There are a few limitations innate to the testing protocol. Failure within our test setup does not have to result in failure in the clinical setup. The testing setup was devised solely to analyse construct stiffness, ignoring additional factors like intact ligaments, soft tissue, or pelvic contents that might contribute to overall stability. The upside of focusing on the construct is that we isolate the effect of different configurations on stiffness. The influence of pin anchoring strength in the bone as a possible confounding factor was avoided in this experimental setup, as all tests were conducted with the same pins anchored in the same polyoxymethylene cylinders. Rod configuration by itself should not have an impact on pin anchoring strength. One limitation might be that the pin-rod interfaces were not tightened by use of a torque spanner, but the same person (S.T), applying maximal force. However we are confident that this did not influence the results as no interface failure occurred and difference in stiffness was due to elastic deformation within the rods and pins rather than interface loosening. It is possible that a bulkier configuration would be prone to screw loosening, being more difficult to handle in postop care. An additional limitation is that forces on the pelvis in real patients are not limited to those addressed in this study. It is possible that the construct and its configurations react differently to a combination of translational and rotational forces or a completely different force vector.

There might be other configurations with better biomechanical properties than those tested in our study. Based on the data of the five tested, however, it seems that where the rod was in parallel alignment with the applied force (for the compression/distraction part), the stiffness was greatest. Moreover where an additional rod could be applied, a significant increase in translational stiffness was recorded. Therefore, when the circumstances (patient size, concomitant injuries, etc.) allow it, one should always choose a configuration where the rod is aligned parallel to the expected force vector. If possible the configuration should be augmented with an extra rod, set up the same way as the first one. The study has shown that the "DOUBLE TENT" configuration is significantly less stiff than the "DOUBLE" one. It is not possible to tell from this study whether a "TENT" configuration provides sufficient stability in obese patients in the clinical environment as obesity is also associated with higher loads. An alternative might be a subcutaneous internal anterior fixator, with which the distance from pin entry sites to the connecting rods can be decreased $[25,26]$. This method would also be an option where further abdominal surgeries are expected avoiding a potentially unstable "RHOMBOID" configuration. There is some evidence that such a device would be at least as stable as an external fixator [27].

Any future studies should look at failure of different constructs in the clinical setup and try to come up with quantifiable, maybe in vivo, results.

\section{Conclusion}

Using two parallel connecting rods for external pelvic ring fixation provides the highest translational (lateral compression/distraction) and rotational (bending of the hip) stability. 


\section{Conflict of Interests}

The authors declare that there is no conflict of interests regarding the publication of this paper.

\section{References}

[1] Z. Balogh, K. L. King, P. Mackay et al., "The epidemiology of pelvic ring fractures: a population-based study," Journal of Trauma, vol. 63, no. 5, pp. 1066-1073, 2007.

[2] D. J. Hak, W. R. Smith, and T. Suzuki, "Management of hemorrhage in life-threatening pelvic fracture," Journal of the American Academy of Orthopaedic Surgeons, vol. 17, no. 7, pp. 447-457, 2009.

[3] M. Tile, "Pelvic ring fractures: should they be fixed?" Journal of Bone and Joint Surgery B, vol. 70, no. 1, pp. 1-12, 1988.

[4] M. C. Moss and M. D. Bircher, "Volume changes within the true pelvis during disruption of the pelvic ring-where does the haemorrhage go?" Injury, vol. 27, no. 1, pp. SA21-SA23, 1996.

[5] A. R. Burgess, B. J. Eastridge, J. W. R. Young et al., "Pelvic ring disruptions: effective classification system and treatment protocols," Journal of Trauma, vol. 30, no. 7, pp. 848-856, 1990.

[6] M. R. Grimm, M. S. Vrahas, and K. A. Thomas, "Pressurevolume characteristics of the intact and disrupted pelvic retroperitoneum," Journal of Trauma, vol. 44, no. 3, pp. 454-459, 1998.

[7] B. L. Riemer, S. L. Butterfield, D. L. Diamond et al., "Acute mortality associated with injuries to the pelvic ring: the role of early patient mobilization and external fixation," Journal of Trauma, vol. 35, no. 5, pp. 671-677, 1993.

[8] P. R. Miller, P. S. Moore, E. Mansell et al., "External fixation or arteriogram in bleeding pelvic fracture: initial therapy guided by markers of arterial hemorrhage," Journal of Trauma, vol. 54, no. 3, pp. 437-443, 2003.

[9] C. Arvieux, F. Thony, C. Broux et al., "Current management of severe pelvic and perineal trauma," Journal of Visceral Surgery, vol. 149, pp. e227-e238, 2012.

[10] W. Chen, Z. Hou, Y. Su et al., "Treatment of posterior pelvic ring disruptions using a minimally invasive adjustable plate," Injury, vol. 44, no. 7, pp. 975-980, 2013.

[11] M. D. Bircher, "Indications and techniques of external fixation of the injured pelvis," Injury, vol. 27, Supplement 1, pp. 3-19, 1996.

[12] M. Scaglione, P. Parchi, G. Digrandi, M. Latessa, and G. Guido, "External fixation in pelvic fractures," Musculoskeletal Surgery, vol. 94, no. 2, pp. 63-70, 2010.

[13] W.-Y. Kirn, T. C. Hearn, O. Seleem, E. Mahalingam, D. Stephen, and M. Tile, "Effect of pin location on stability of pelvic external fixation," Clinical Orthopaedics and Related Research, no. 361, pp. 237-244, 1999.

[14] M. J. Gardner and S. E. Nork, "Stabilization of unstable pelvic fractures with supraacetabular compression external fixation," Journal of Orthopaedic Trauma, vol. 21, no. 4, pp. 269-273, 2007.

[15] D. Demetriades, M. Karaiskakis, K. Toutouzas, K. Alo, G. Velmahos, and L. Chan, "Pelvic fractures: epidemiology and predictors of associated abdominal injuries and outcomes," Journal of the American College of Surgeons, vol. 195, no. 1, pp. 1-10, 2002.

[16] W. D. Dupont and W. D. Plummer Jr., "Power and sample size calculations for studies involving linear regression," Controlled Clinical Trials, vol. 19, no. 6, pp. 589-601, 1998.
[17] J. J. Wild Jr., G. W. Hanson, and H. S. Tullos, "Unstable fractures of the pelvis treated by external fixation," Journal of Bone and Joint Surgery A, vol. 64, no. 7, pp. 1010-1020, 1982.

[18] T. Pohlemann, C. Krettek, R. Hoffmann, U. Culemann, and A. Gansslen, "Biomechanical comparison of different devices for emergency stabilization of the pelvis," Unfallchirurg, vol. 97, no. 10, pp. 503-510, 1994.

[19] M. C. Tucker, S. E. Nork, P. T. Simonian, and M. L. Chip Routt Jr., "Simple anterior pelvic external fixation," Journal of Trauma, vol. 49, no. 6, pp. 989-994, 2000.

[20] M. T. Archdeacon, S. Arebi, T. T. Le, R. Wirth, R. Kebel, and M. Thakore, "Orthogonal pin construct versus parallel uniplanar pin constructs for pelvic external fixation: a biomechanical assessment of stiffness and strength," Journal of Orthopaedic Trauma, vol. 23, no. 2, pp. 100-105, 2009.

[21] V. Vecsei, "Results of biomechanical examinations of various external fixation mountings in the pelvic region," Aktuelle Traumatologie, vol. 18, no. 6, pp. 261-264, 1988.

[22] H.-J. Egbers, F. Draijer, D. Havemann, and W. Zenker, "Stabilisation of the pelvic girdle by external fixation. Biomechanical investigations and clinical experience," Orthopade, vol. 21, no. 6, pp. 363-372, 1992.

[23] H. Rieger, S. Winckler, D. Wetterkamp, and J. Overbeck, "Clinical and biomechanical aspects of external fixation of the pelvis," Clinical Biomechanics, vol. 11, no. 6, pp. 322-327, 1996.

[24] K. J. Ponsen, G. A. Hoek Van Dijke, P. Joosse, and C. J. Snijders, "External fixators for pelvic fractures: comparison of the stiffness of current systems," Acta Orthopaedica Scandinavica, vol. 74, no. 2, pp. 165-171, 2003.

[25] M. J. Gardner, S. Mehta, A. Mirza, and W. M. Ricci, "Anterior pelvic reduction and fixation using a subcutaneous internal fixator," Journal of Orthopaedic Trauma, vol. 26, no. 5, pp. 314-321, 2012.

[26] R. Vaidya, R. Colen, J. Vigdorchik, F. Tonnos, and A. Sethi, "Treatment of unstable pelvic ring injuries with an internal anterior fixator and posterior fixation: initial clinical series," Journal of Orthopaedic Trauma, vol. 26, no. 1, pp. 1-8, 2012.

[27] J. M. Vigdorchik, A. O. Esquivel, X. Jin et al., "Biomechanical stability of a supra-acetabular pedicle screw internal fixation device (INFIX) vs external fixation and plates for vertically unstable pelvic fractures," Journal of Orthopaedic Surgery and Research, vol. 7, article 31, 2012. 


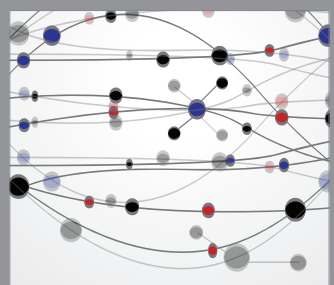

The Scientific World Journal
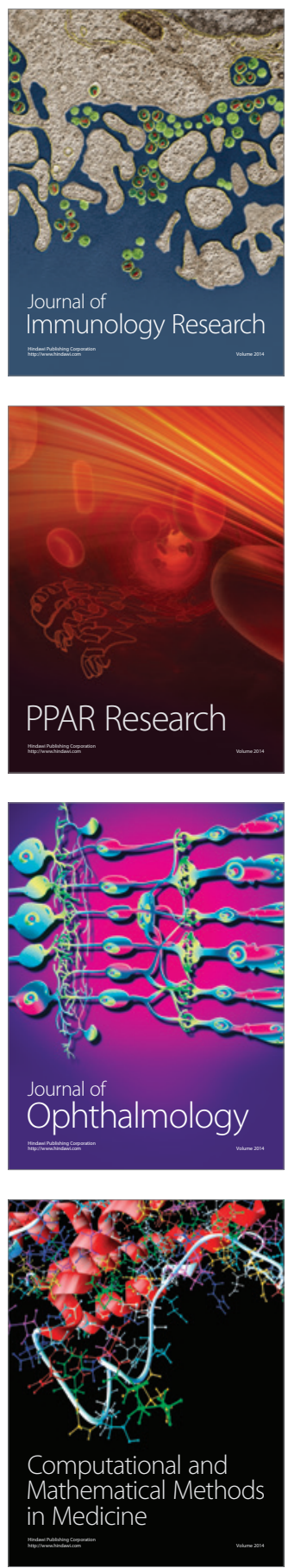

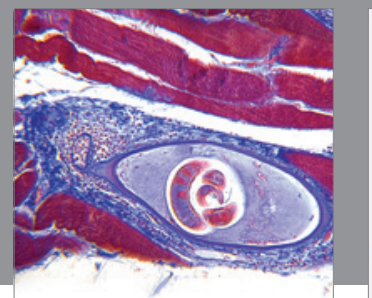

Gastroenterology

Research and Practice
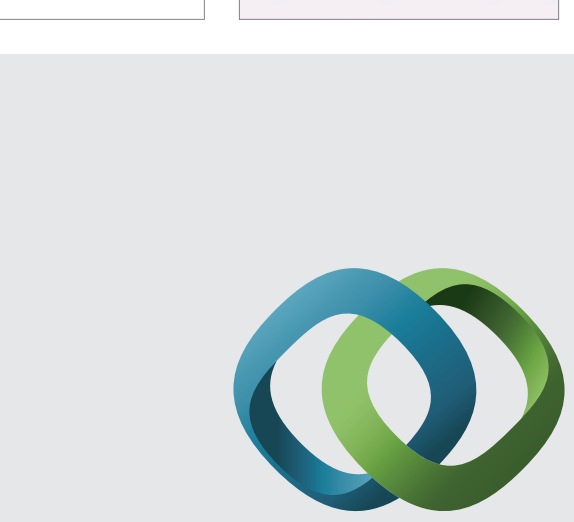

\section{Hindawi}

Submit your manuscripts at

http://www.hindawi.com
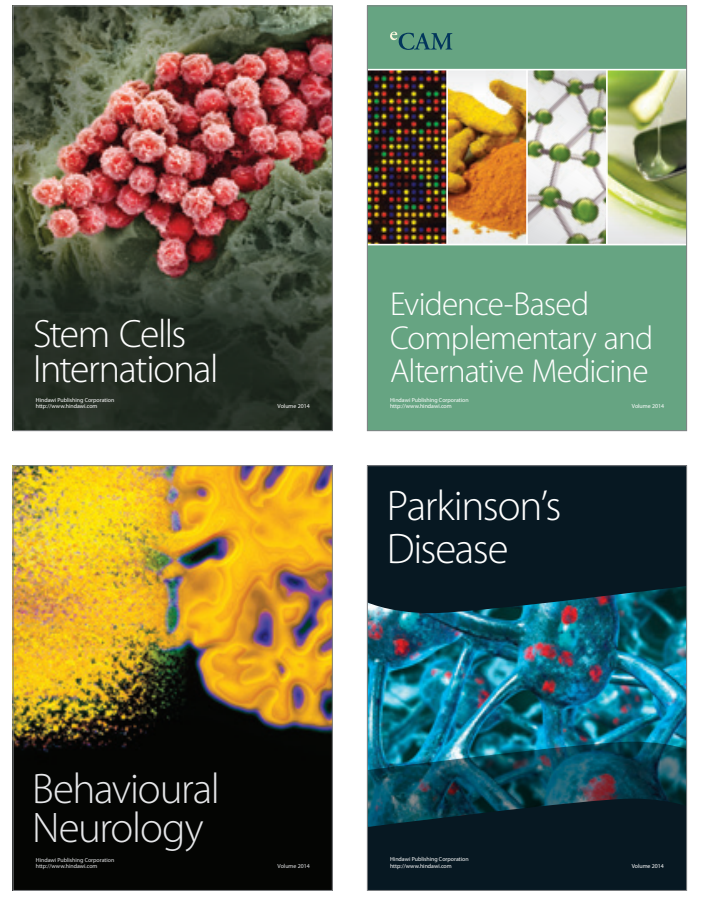
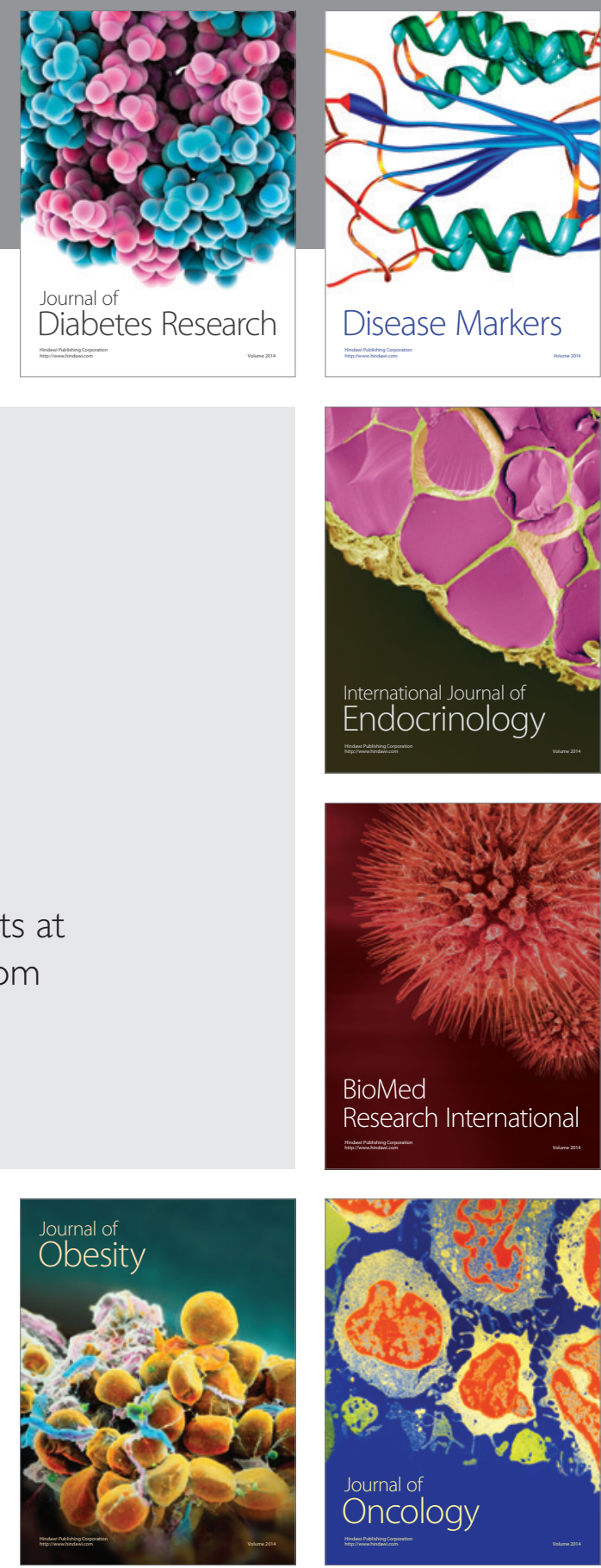

Disease Markers
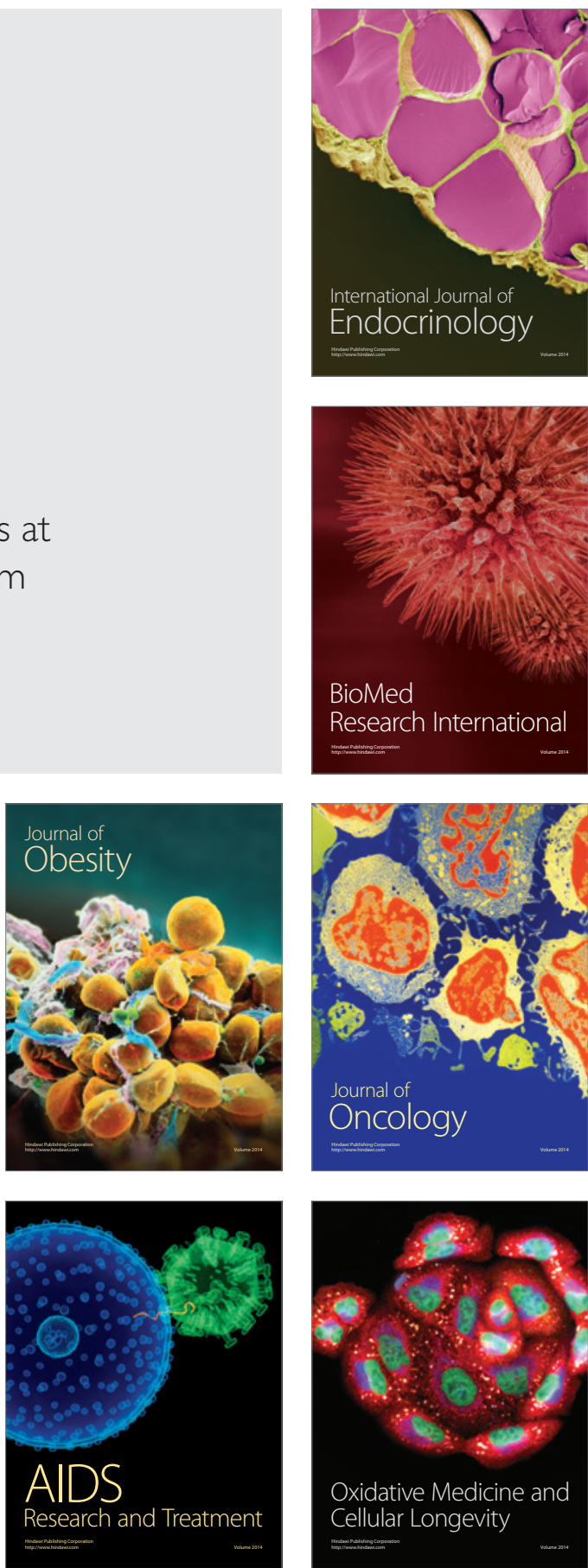